\title{
A Rare Case of Coexisting Superior Mesenteric Artery Syndrome and Nutcracker Phenomenon
}

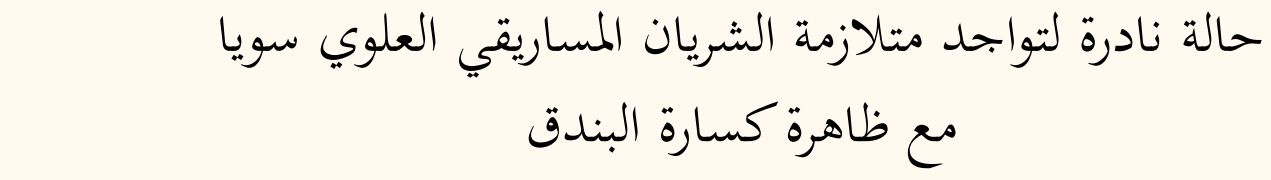

فيليبس جورج مايكل، طاهرة السعدية، راكيش جامخانديكار
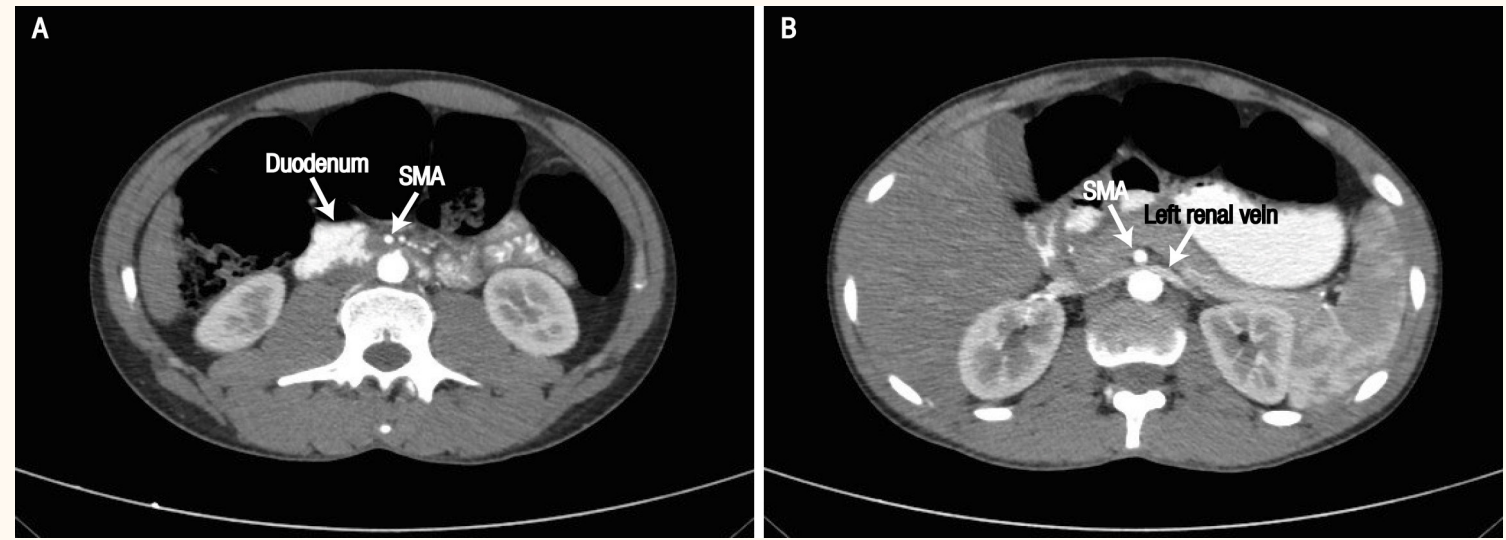

Figure 1: Axial contrast-enhanced computed tomography scans of the abdomen of a 28-year-old man showing (A) duodenal compression between the superior mesenteric artery (SMA) and abdominal aorta and (B) impingement of the left renal vein by the SMA.

SMA = superior mesenteric artery.

A 28-YEAR-OLD MAN WAS REFERRED AS AN emergency case from a regimental medical clinic to the surgical unit of the Armed Forces Hospital, Muscat, Oman, in 2016 with complaints of severe vomiting, epigastric pain and bloating of two days' duration. The patient gave a history of abdominal bloating associated with early satiety, postprandial pain and occasional vomiting over the past year and a half. There was no other significant medical or surgical history. Clinically, the patient was underweight and pale with a body mass index of $17 \mathrm{~kg} / \mathrm{m}^{2}$. An abdominal examination revealed upper abdominal tenderness and distention. The findings of initial blood tests and laboratory investigations were unremarkable. An ultrasound of the abdomen performed two days prior at the previous institution demonstrated no sonographical abnormalities.

Due to the patient's long-standing symptoms and weight loss, a contrast-enhanced computed tomo- graphy (CT) scan of the abdomen was performed using a Philips Brilliance 64-slice CT scanner (Philips Healthcare, Cleveland, Ohio, USA). This revealed a distended stomach and dilated proximal duodenum with narrowing of the third part of the duodenum between the angle of the superior mesenteric artery (SMA) and the abdominal aorta, with a shortened aortomesenteric distance (AMD) of $7 \mathrm{~mm}$ and a decreased aortomesenteric angle (AA) of 15 degrees. In addition, the left renal vein was compressed by the SMA close to its origin [Figures 1 and 2]. Based on these imaging findings, a diagnosis of SMA syndrome and nutcracker phenomenon was made. Upon further questioning, the patient gave a history of intentional dieting and weight loss of approximately $15 \mathrm{~kg}$ over the last two years in order to meet the fitness criteria for military recruitment; subsequently, he had continued his dietary regimen even after being recruited into the army. However, he denied experiencing any 


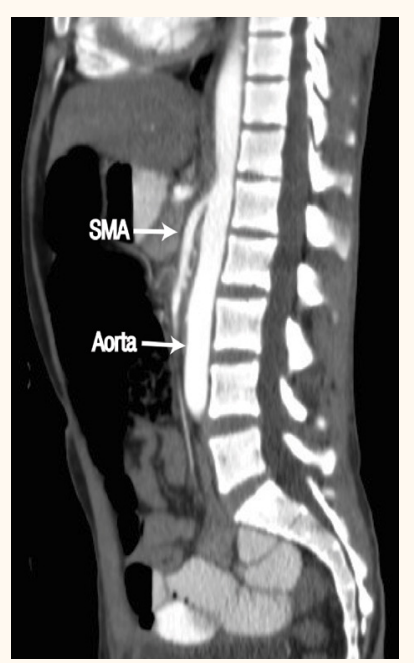

Figure 2: Sagittal contrast-enhanced computed tomography scan of the abdomen of a 28-year-old man demonstrating the acute angle of the superior mesenteric artery at its point of origin from the abdominal aorta, with a decreased aortomesenteric distance.

SMA = superior mesenteric artery.

haematuria and a second clinical examination did not reveal any left-sided varicoceles.

The patient was managed conservatively with nutritional support to encourage weight gain via combined total parenteral and enteral nutrition. Multidisciplinary treatment with input from general surgery, gastroenterology and dietetic experts was effective in reducing his epigastric abdominal pain. The patient also underwent a gastroduodenoscopy which did not reveal any intrinsic obstructions. Since he had normal renal function parameters with normal blood pressure, no further intervention was deemed necessary to treat the nutcracker phenomenon. The patient was discharged and subsequently had an uneventful recovery.

\section{Comment}

The extrinsic compression of the third part of the duodenum between the SMA and the abdominal aorta as the artery crosses the duodenum anteriorly-known as SMA syndrome-is a rare cause of upper gastrointestinal obstruction. ${ }^{1}$ Patients may present with chronic or vague postprandial symptoms, such as upper abdominal pain, nausea, vomiting, weight loss and a feeling of early satiety or bloating, although acute presentations with symptoms of duodenal obstruction have also been described. ${ }^{1,2}$ The syndrome occurs due to an abnormally acute angle between the proximal part of the SMA and the abdominal aorta which ranges from 6-16 degrees in symptomatic patients compared to $38-56$ degrees in normal individuals; this results in duodenal compression and a reduced mean AMD of 2-8 $\mathrm{mm}$ in symptomatic patients compared to normal mean values of $10-28 \mathrm{~mm}^{3}$ Abnormally steep angulation between the SMA and the aorta can cause a short AMD and predispose to nutcracker syndrome. ${ }^{2}$ A significantly decreased AMD of $3 \mathrm{~mm}$ and decreased AA of $<15$ degrees has been previously reported in patients with nutcracker syndrome..$^{2-4}$

A decreased AA may be due to rapid weight loss resulting in reduced fat and lymphatic tissue at the origin of the SMA, decreasing the ability to cushion the duodenum and protect it from compression., ${ }^{2,3}$ In the current case, the patient's significant weight loss, particularly of intra-abdominal fat, was thought to be a contributing factor to the unusual vascular compression of the duodenum. Other factors can include anatomical abnormalities such as a short ligament of Treitz, pressure from external sources such as body casts and postoperative complications from procedures such as abdominal aortic aneurysm repairs, scoliosis surgeries, ileo-anal pouch anastomoses and total proctocolectomies., ${ }^{3,4}$ For most patients with SMA syndrome, conservative management-including the correction of electrolyte imbalances along with appropriate enteral or parenteral nutrition-usually results in sufficient weight gain and symptomatic improvement. ${ }^{3}$ Surgical procedures such as a duodenojejunostomy or the surgical release of the Treitz ligament are reserved for cases which do not respond to conservative treatment. ${ }^{2-4}$

Nutcracker phenomenon refers to the compression of the left renal vein between the SMA and the abdominal aorta., ${ }^{2,3}$ Affected patients may be asymptomatic or present with haematuria, proteinuria or left-sided flank pain or varicoceles. ${ }^{1-3}$ Conservative management is often sufficient for symptomatic patients, although endovascular stenting is the treatment of choice if necessary. ${ }^{4}$ Although the terms 'nutcracker syndrome' and 'nutcracker phenomenon' are sometimes used interchangeably, some symptoms may be unassociated and certain anatomical findings may represent a normal variant; hence, the term 'nutcracker syndrome' should be reserved for patients with characteristic clinical symptoms associated and demonstrable morphological features. ${ }^{2-4}$

Besides the classic (i.e. anterior) nutcracker syndrome, other anatomical variants have been described. ${ }^{4,5}$ Posterior nutcracker syndrome involves compression of the left renal vein between the abdominal aorta and vertebral spine, whereas combined nutcracker syndrome refers to a duplication of the vein causing compression of either the anterior branch between the abdominal aorta and SMA or posterior branch between the abdominal aorta and 
spine. ${ }^{3,4}$ Lateral or anterolateral nutcracker phenomenon describes venous compression as the vein passes between the SMA and the right renal artery. ${ }^{5}$ Clinically, nutcracker syndrome has been differentiated into the typical subtype with renal manifestations such as haematuria and proteinuria with or without flank pain and the atypical subtype involving urological manifestations such as fatigue, flank/abdominal pain, orthostatic intolerance and dysmenorrhoea and dyspareunia in women and varicoceles in men..$^{2-5}$

Both nutcracker phenomenon and SMA syndrome are rare vascular compression entities and CT findings of a decreased AA and shortened AMD are suggestive of a diagnosis for both conditions. ${ }^{1-5}$ However, clinical symptoms may not correlate with the severity of the anatomical distortion, even in extreme cases, thus making it challenging to diagnose..$^{3-5}$ Very few coexisting cases have been described in the existing medical literature. ${ }^{2-5}$ Clinicians and radiologists should therefore be aware of and consider these conditions in cases of unexplained abdominal pain without corresponding biochemical or clinical findings.

\section{References}

1. Inal M, Unal Daphan B, Karadeniz Bilgili MY. Superior mesenteric artery syndrome accompanying with nutcracker syndrome: A case report. Iran Red Crescent Med J 2014; 16:e14755. doi: 10.5812/ircmj.14755.

2. Barsoum MK, Shepherd RF, Welch TJ. Patient with both Wilkie syndrome and nutcracker syndrome. Vasc Med 2008; 13:247-50. doi: 10.1177/1358863X08092272.

3. Karaosmanoğlu D, Karcaaltincaba M, Akata D, Ozmen M. Unusual causes of left renal vein compression along its course: MDCT findings in patients with nutcracker and pelvic congestion syndrome. Surg Radiol Anat 2010; 32:323-7. doi: 10.1007/s00276-009-0548-1.

4. Nunn R, Henry J, Slesser AA, Fernando R, Behar N. A model example: Coexisting superior mesenteric artery syndrome and the nutcracker phenomenon. Case Rep Surg 2015; 2015:649469. doi: $10.1155 / 2015 / 649469$.

5. Polguj M, Topol M, Majos A. An unusual case of left venous renal entrapment syndrome: A new type of nutcracker phenomenon? Surg Radiol Anat 2013; 35:263-7. doi: 10.1007/ s00276-012-1027-7. 